\title{
三氟甲磺酸酯的高效合成
}

\author{
郑东青 $a, b$ 马海燕*,b 丁 凯*,a \\ $\left({ }^{a}\right.$ 中国科学院上海有机化学研究所 天然产物合成有机化学重点实验室 上海 200032) \\ ${ }^{b}$ 华东理工大学化学系 上海 200237)
}

\begin{abstract}
摘要 原甲酸酯与三氟甲磺酸酐反应可高效合成三氟甲磺酸酯. 该方法具有无需溶剂, 反应条件温和, 反应时间短, 高产率, 操作简单, 底物范围广的特点. 原位产生的试剂具有高的反应活性, 可一锅法高产率制备三氟甲磺酸离子液 体.

关键词 三氟甲磺酸酯; 原酸酯; 三氟甲磺酸䣶; 一锅法烷基化
\end{abstract}

\section{A Practical Synthesis of Trifluoromethanesulfonate Esters}

\author{
Zheng, Dongqing ${ }^{a, b}$ Ma, Haiyan ${ }^{*, b}$ Ding, Kai*,a \\ $\left({ }^{a}\right.$ CAS Key Laboratory of Synthetic Chemistry of Natural Substances, Shanghai Institute of Organic Chemistry, \\ Chinese Academy of Sciences, Shanghai 200032) \\ ( $b$ Department of Chemistry, East China University of Science and Technology, Shanghai 200237)
}

\begin{abstract}
A practical synthesis of trifluoromethanesulfonate esters by reaction of orthoformate esters with triflic anhydride is described. The solvent-free method featured mild condition, short time, high yield, simple operation and broad substrate scope. The in situ generated trifluoromethanesulfonate ester is highly reactive alkylating agent, providing triflate ionic liquid in excellent yield via a one-pot procedure.

Keywords trifluoromethanesulfonate ester; orthoester; triflic anhydride; one-pot alkylation
\end{abstract}

Trifluoromethanesulfonate esters (triflate esters) are versatile alkylating agent and broadly used in synthetic organic chemistry. ${ }^{[1]}$ However, only a very small number of these regents are commercially available due to their instability. Therefore, many synthetic methods have been developed for the synthesis of triflate esters in the laboratory (Scheme 1). ${ }^{[2 \sim 6]}$

Pure triflate esters were first synthesised by the reaction of silver triflate with alkyl halide. ${ }^{[2]}$ The method is clearly not economical and was soon replaced with the reaction of triflic anhydride and alcohol in the presence of acid scavengers. ${ }^{3}$ Although the method is widely used, removal of solvents and by-products from unstable products are often inefficient and laborious. Begue et al. ${ }^{[4]}$ developed a simple method for the preparation of triflate esters by reaction of alkyl trimethylsilyl ethers with triflic anhydride. The low boiling by-product TMSOTf is easily removed by distillation. But the method is not economical because half of the triflic anhydride is lost. The reaction of triflic anhydride with dialkyl sulphate ${ }^{[5]}$ or dialkyl carbonate ${ }^{[6]}$ over- a) the reaction of silver triflate with alkyl halides

AgOTf $+\mathrm{RX} \longrightarrow$ ROTf + AgX

b) the reaction of triflic anhydride with alcohols

$$
\mathrm{Tf}_{2} \mathrm{O}+\mathrm{ROH} \stackrel{\text { base }}{\longrightarrow} \text { ROTf + base } \cdot \mathrm{TfOH}
$$

c) the reaction of triflic acid or triflic anhydride with esters $\mathrm{Tf}_{2} \mathrm{O}+\mathrm{ROSiMe}_{3} \stackrel{0^{\circ} \mathrm{C}}{\longrightarrow} \mathrm{ROTf}+\mathrm{TMSOTf}$

d) the reaction of triflic acid or triflic anhydride with esters

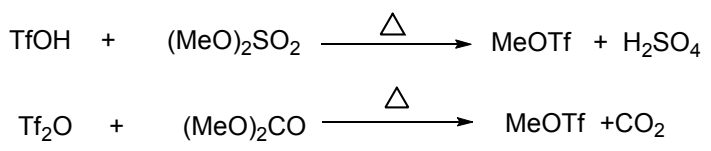

Scheme 1 Traditional synthesis of triflate esters

came the drawback. However, these methods required high reaction temperature and consequently suffered from a limited substrate scope. Herein, we report a practical method for the preparation of triflate esters by reaction of

\footnotetext{
* Corresponding authors. E-mail: haiyanma@ecust.edu.cn; dingkai@mail.sioc.ac.cn Received January 18, 2017; revised and published online January 20, 2017.

Project supported by the National Natural Science Foundation of China (No. 20902098).

国家自然科学基金(No. 20902098)资助项目.
} 
orthoformate esters with triflic anhydride. The solvent-free method featured mild condition $\left(0 \sim 25{ }^{\circ} \mathrm{C}\right)$, short time $(15$ min), high yield (up to $99 \%$ ), simple operation and broad substrate scope.

\section{Results and discussion}

During the course of our studies on the synthesis of steroidal estrogens, ${ }^{[7]} \mathrm{TfOH}$ as a catalyst was deactivated in the presence of orthoformate esters even at room temperature, which was rationalized by the formation of triflate esters. ${ }^{[8]}$ However, the attempt to prepare methyl triflate from trimethyl orthoformate was unsuccessful due to subsequent etherification with methanol. We presumed that using triflic anhydride instead of TfOH would scavenge the alcohol and suppress the undesired etherification. As expected, the reaction of triflic anhydride and methyl orthoformate provided methyl triflate in high yield at room temperature only with a trace amount of dimethyl ether (Table 1, Entry 1). Initial attempt to suppress the etherification by lowering the reaction temperature was unsuccessful (Entry 2). Fortunately, changing the addition order of reagents solved the problem because the excess $\mathrm{Tf}_{2} \mathrm{O}$ minimized the concentration of alcohol (Entry 3). The highly exothermic reaction is self-accelerating. To prevent the loss of low boiling reagents and products, the reaction was initiated at low temperature and performed at room temperature.

Table 1 Optimization of condition ${ }^{a}$

\begin{tabular}{|c|c|c|c|c|}
\hline Entry $^{a}$ & Temp. $/{ }^{\circ} \mathrm{C}$ & Order $^{b}$ & Time/min & Yield $^{c} \%$ \\
\hline 1 & 25 & A & $<5$ & $95^{d}$ \\
\hline 2 & 0 & A & 120 & $83^{d, e}$ \\
\hline 3 & $0 \sim 25$ & B & 15 & 100 \\
\hline
\end{tabular}

${ }^{a} \mathrm{Tf}_{2} \mathrm{O}(6 \mathrm{mmol})$, orthoester $(6 \mathrm{mmol}) ;{ }^{b} \mathrm{~A}$ : add $\mathrm{Tf}_{2} \mathrm{O}$ into orthoester, $\mathrm{B}$ : add orthoester into $\mathrm{Tf}_{2} \mathrm{O} ;{ }^{c} \mathrm{NMR}$ yield; ${ }^{d}$ dimethyl ether was found $(\approx 5 \%){ }^{e}{ }^{e}$ conversion.

Encouraged by the result, the substrate scope was investigated. Orthoformate esters were prepared from alcohols, formamide and benzoyl chloride. ${ }^{[9]}$ Triflate esters were smoothly synthesized in excellent yield according to above procedure (Table 2, Entries $1 \sim 6$ ). The only by- products are formate esters, which can be easily removed by distillation. Notably, the method can provide pure $i-\mathrm{C}_{3} \mathrm{H}_{7}$ - OTf as a colorless liquid though the secondary triflate ester was very unstable and decomposed at room temperature after several hours (Entry 6).

The possible mechanism of the reaction can be described as follow (Scheme 2). In the first step, a trace amount of triflic acid in triflic anhydride reacts with the orthoformate ester to provide alcohol, formate ester and triflic ester. Subsequently, triflic anhydride plays a role of alcohol scavenger to regenerate the triflic acid.

Triflate ester is powerful alkylating agent and, in most cases, can react with nucleophiles under mild condition, which is inert to carboxylic ester. Therefore, a one-pot, two-
Table 2 Substrates scope ${ }^{a}$

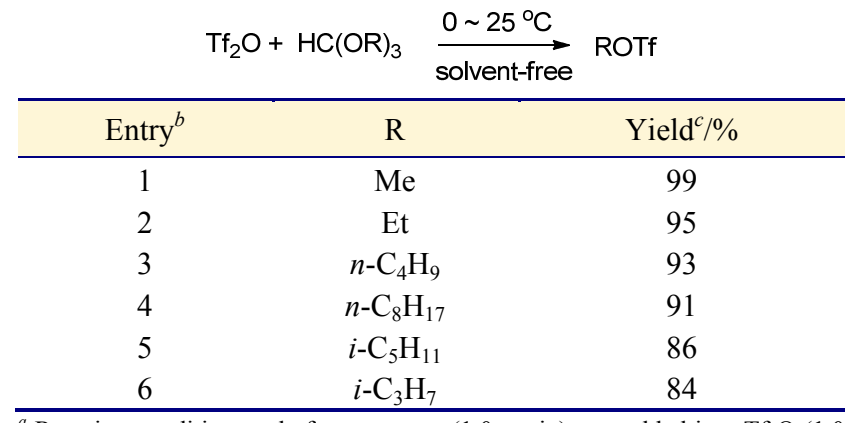

${ }^{a}$ Reaction condition: orthoformate ester (1.0 equiv) was added into $\mathrm{Tf}_{2} \mathrm{O}(1.0$ equiv), $0 \sim 25{ }^{\circ} \mathrm{C}$ for $15 \mathrm{~min} .{ }^{b}$ Entries $1 \sim 2$ on $30 \mathrm{mmol}$ scale; Entries $3 \sim 7$ on 15 mmol scale. ${ }^{b}$ Isolated yield.

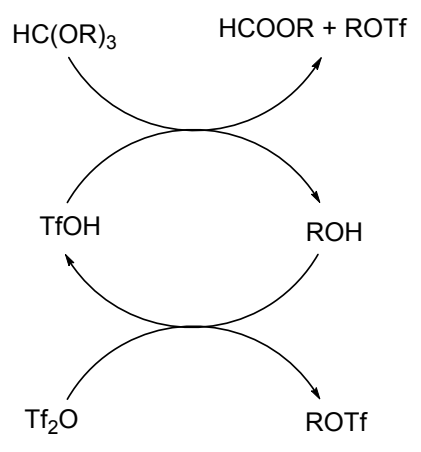

Scheme 2 Possible mechanism

step protocol that bypasses the purification of the unstable and toxic alkylating agents would be possible. Triflate ionic liquid was efficiently synthesized using the protocol on 30 mmol scale (Scheme 3). The only by-product was low boiling formate ester, which was easily removed under reduced pressure to provide highly pure ionic liquid.

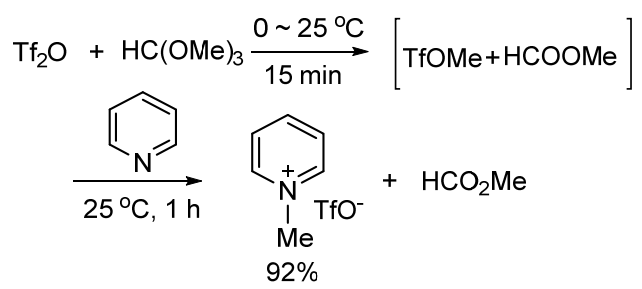

Scheme 3 One-pot synthesis of triflate ionic liquid

\section{Conclusions}

In this paper, we describe a practical synthesis of triflic esters from easily available trifilc anhydride and orthoformate esters under mild condition in excellent yield. Based on the method, a one-pot, two-step alkylation protocol was applied to synthesize triflate ionic liquid.

\section{Experimental}

All NMR experiments were recorded on Agilent 400 $\mathrm{MHz}$ NMR spectrometer or Bruker $400 \mathrm{MHz}$ NMR spectrometer. Chemical shifts referenced to the residual solvent peak $\left({ }^{1} \mathrm{H} \mathrm{7.26},{ }^{13} \mathrm{C} 77.0\right.$ for $\left.\mathrm{CDCl}_{3}\right)$. Tf $2 \mathrm{O}$, trimethyl orthoformate and triethyl orthoformate were commercially 
available and used after distillation under reduced pressure. Other orthoformates were synthesized according to the reported method. ${ }^{[9]}$

General procedure for synthesis of triflate ester: Orthoformate ester (1.0 equiv) was added into $\mathrm{Tf}_{2} \mathrm{O}(1.0$ equiv) at $0{ }^{\circ} \mathrm{C}$. The mixture was stirred at room temperature for $15 \mathrm{~min}$. The pure product was obtained after a distillation under reduced pressure.

Methyl trifluorosulfonate ${ }^{[6]}$ : b.p. $47{ }^{\circ} \mathrm{C} / 5.6 \mathrm{kPa} ;{ }^{1} \mathrm{H}$ NMR $\left(400 \mathrm{MHz}, \mathrm{CDCl}_{3}\right) \delta: 4.21(\mathrm{~s}, 3 \mathrm{H}) ;{ }^{13} \mathrm{C} \mathrm{NMR}(101 \mathrm{MHz}$, $\left.\mathrm{CDCl}_{3}\right) \delta: 118.7(\mathrm{q}, J=321.2 \mathrm{~Hz}), 61.6 ;{ }^{19} \mathrm{~F}$ NMR $(376$ $\left.\mathrm{MHz}, \mathrm{CDCl}_{3}\right) \delta:-74.5$ (s, 3F).

Ethyl trifluorosulfonate ${ }^{[6]}$ : b.p. $42{ }^{\circ} \mathrm{C} / 5.6 \mathrm{kPa} ;{ }^{1} \mathrm{H}$ NMR $\left(400 \mathrm{MHz}, \mathrm{CDCl}_{3}\right) \delta: 4.62(\mathrm{q}, J=7.1 \mathrm{~Hz}, 2 \mathrm{H}), 1.51(\mathrm{t}, J=$ $7.1 \mathrm{~Hz}, 3 \mathrm{H}) ;{ }^{13} \mathrm{C}$ NMR $\left(101 \mathrm{MHz}, \mathrm{CDCl}_{3}\right) \delta: 118.6$ (q, $J=$ $320.8 \mathrm{~Hz}), 74.0,15.2 ;{ }^{19} \mathrm{~F}$ NMR $\left(376 \mathrm{MHz}, \mathrm{CDCl}_{3}\right) \delta:-$ $75.2(\mathrm{~s}, 3 \mathrm{~F})$.

n-Butyl trifluorosulfonate ${ }^{[4]}$ : b.p. $40{ }^{\circ} \mathrm{C} / 0.5 \mathrm{kPa} ;{ }^{1} \mathrm{H}$ NMR $\left(400 \mathrm{MHz}, \mathrm{CDCl}_{3}\right) \delta: 4.54(\mathrm{t}, J=6.4 \mathrm{~Hz}, 2 \mathrm{H}), 1.87 \sim 1.74$ $(\mathrm{m}, 2 \mathrm{H}), 1.47(\mathrm{dd}, J=15.0,7.5 \mathrm{~Hz}, 2 \mathrm{H}), 0.97(\mathrm{t}, J=7.4 \mathrm{~Hz}$, $3 \mathrm{H}) ;{ }^{13} \mathrm{C}$ NMR (101 MHz, $\left.\mathrm{CDCl}_{3}\right) \delta: 118.6$ (q, $J=320.8$ $\mathrm{Hz}), 77.5,31.1,18.3,13.1 ;{ }^{19} \mathrm{~F} \mathrm{NMR}\left(376 \mathrm{MHz}, \mathrm{CDCl}_{3}\right) \delta$ : $-75.3(\mathrm{~s}, 3 \mathrm{~F})$.

$n$-Heptyl trifluorosulfonate ${ }^{[4]}$ : b.p. $48 \sim 50{ }^{\circ} \mathrm{C} / 27 \mathrm{~Pa} ;{ }^{1} \mathrm{H}$ NMR (400 MHz, $\left.\mathrm{CDCl}_{3}\right) \delta: 4.53(\mathrm{t}, J=6.5 \mathrm{~Hz}, 2 \mathrm{H}), 1.90 \sim$ $1.76(\mathrm{~m}, 2 \mathrm{H}), 1.44 \sim 1.28(\mathrm{~m}, 10 \mathrm{H}), 0.89(\mathrm{t}, J=6.8 \mathrm{~Hz}, 3 \mathrm{H})$; ${ }^{13} \mathrm{C}$ NMR (101 MHz, $\left.\mathrm{CDCl}_{3}\right) \delta: 118.7$ (q, $\left.J=321.2 \mathrm{~Hz}\right)$, $77.8,31.7,29.2,29.0,28.8,25.0,22.6,13.9 ;{ }^{19} \mathrm{~F}$ NMR (376 $\left.\mathrm{MHz}, \mathrm{CDCl}_{3}\right) \delta:-75.3(\mathrm{~s}, 3 \mathrm{~F})$.

$i$-Pentyl trifluorosulfonate ${ }^{[10]}$ : b.p. $25^{\circ} \mathrm{C} / 27 \mathrm{~Pa} ;{ }^{1} \mathrm{H}$ NMR $\left(400 \mathrm{MHz}, \mathrm{CDCl}_{3}\right) \delta: 4.57(\mathrm{t}, J=6.5 \mathrm{~Hz}, 2 \mathrm{H}), 1.82 \sim 1.75$ (m, 1H), 1.72 (q, $J=6.5 \mathrm{~Hz}, 2 \mathrm{H}), 0.96(\mathrm{~d}, J=6.4 \mathrm{~Hz}, 6 \mathrm{H})$; ${ }^{13} \mathrm{C}$ NMR $\left(101 \mathrm{MHz}, \mathrm{CDCl}_{3}\right) \delta$ : $118.6(\mathrm{q}, J=321.2 \mathrm{~Hz})$, 76.3, 37.7, 24.4, 22.0; ${ }^{19} \mathrm{~F}$ NMR (376 MHz, $\left.\mathrm{CDCl}_{3}\right) \delta:-$ 75.0 (s, 3F).

$i$-Propyl trifluorosulfonate ${ }^{[4]}$ : b.p. $25{ }^{\circ} \mathrm{C} / 133 \mathrm{~Pa} ;{ }^{1} \mathrm{H}$ NMR $\left(400 \mathrm{MHz}, \mathrm{CDCl}_{3}\right) \delta: 5.25 \sim 5.19(\mathrm{~m}, 1 \mathrm{H}), 1.52(\mathrm{~d}, \quad J=$ $6.3 \mathrm{~Hz}, 7 \mathrm{H}) ;{ }^{13} \mathrm{C}$ NMR $\left(101 \mathrm{MHz}, \mathrm{CDCl}_{3}\right) \delta: 118.5$ (q, $J$ $=320.5 \mathrm{~Hz}), 86.4,23.0 ;{ }^{19} \mathrm{~F} \mathrm{NMR}\left(376 \mathrm{MHz}, \mathrm{CDCl}_{3}\right) \delta$ : 75.9 (s, 3F).

Synthesis of $N$-methylpyridinium triflate: Trimethyl orthoformate $(1.64 \mathrm{~mL}, 15 \mathrm{mmol})$ was added into $\mathrm{Tf}_{2} \mathrm{O}(2.52$ $\mathrm{mL}, 15 \mathrm{mmol})$ at $0{ }^{\circ} \mathrm{C}$. After stirring for $15 \mathrm{~min}$ at room temperature, dry pyridine $(2.73 \mathrm{~g}, 30 \mathrm{mmol})$ was slowly added into the mixture at room temperature, which was stirred for $1 \mathrm{~h}$ at room temperature. The moisture was heated to $60{ }^{\circ} \mathrm{C}$ for $1 \mathrm{~h}$ under reduced pressure to provide the pure product as a thick colorless oil $(6.73 \mathrm{~g}, 92 \%)$, which solidified on standing in a refrigerator. ${ }^{1} \mathrm{H}$ NMR $(400 \mathrm{MHz}$, DMSO- $\left.d_{6}\right) \delta: 8.97(\mathrm{~d}, J=5.8 \mathrm{~Hz}, 2 \mathrm{H}), 8.57(\mathrm{t}, J=7.8 \mathrm{~Hz}$, $1 \mathrm{H}), 8.12(\mathrm{t}, J=7.0 \mathrm{~Hz}, 2 \mathrm{H}), 4.36(\mathrm{~s}, 3 \mathrm{H}) ;{ }^{13} \mathrm{C} \mathrm{NMR}(101$ MHz, DMSO- $d_{6}$ ) $\delta: 145.6,145.1,127.8,120.8$ (q, $J=323.5$ $\mathrm{Hz}), 48.0 ;{ }^{19} \mathrm{~F}$ NMR $\left(376 \mathrm{MHz}, \mathrm{DMSO}-d_{6}\right) \delta:-77.8(\mathrm{~s}$, $3 \mathrm{~F})$.

Supporting Information ${ }^{1} \mathrm{H}$ NMR, ${ }^{13} \mathrm{C}$ NMR and ${ }^{19} \mathrm{~F}$ NMR spectra of all compounds. The Supporting Information is available free of charge via the Internet at http://sioc-journal.cn.

\section{References}

[1] (a) Ulibarri, G.; Choret, N.; Bigg, D. C. H. Synthesis 1996, 1286. (b) Wang, S. F.; Zhang, A. J. Org. Prep. Proced. Int. 2008, 40 (3), 293.

(c) Chen, Z. J.; Xi, H. W.; Lim, K. H.; Lee, J. M. Angew. Chem., Int. Ed. 2013, 52(50), 13392.

(d) Dang, H.; Mailig, M.; Lalic, G. Angew. Chem., Int. Ed. 2014, 53(25), 6473.

(e) Zhao, P.; Yan, X. Y.; Yin, H.; Xi, C. J. Org. Lett. 2014, 16(4), 1120 .

[2] (a) Gramstad, T.; Haszeldine, R. N. J. Chem. Soc. 1956, 173.1.

(b) Chapman, R. D.; Andreshak, J. L.; Herrlinger, S. P.; Shackelford, S. A.; Hildreth, R. A.; Smith, J. P. J. Org. Chem. 1986, 51(20), 3792.

[3] Baraznenok, I. L.; Nenajdenko, V. G.; Balenkova, E. S. Tetrahedron 2000, 56(20), 3077.

[4] Aubert, C.; Begue, J. P. Synthesis 1985, (8), 759.

[5] Beard, C. D.; Baum, K.; Grakausk, V. J. Org. Chem. 1973, 38(21), 3673.

[6] Ignat'ev, N. V.; Barthen, P.; Kucheryna, A.; Willner, H.; Sartori, P. Molecules 2012, 17(5), 5319.

[7] Zheng, D.-Q.; Jing, Y.; Zheng, B.-Y.; Ye, Y.-F.; Xu, S.; Tian, W.-S.; Ma, H.-Y.; Ding, K. Tetrahedron 2016, 72(17), 2164.

[8] (a) Padmapriya, A. A.; Just, G.; Lewis, N. G. Synth. Commun. 1985 15(12), 1057.

(b) Trujillo, J. I.; Gopalan, A. S. Tetrahedron Lett. 1993, 34(46), 7355.

(c) Yoshino, T.; Togo, H. Synlett 2005.

[9] Ohme, R.; Schmitz, E. Justus Liebigs Ann. Chem. 1968, 716, 207.

[10] Anderson, G. L.; Harruna, I. Synth. Commun. 1987, 17(1), 111. 